\section{Pleural fluid lysozyme in tuberculous and non-tuberculous pleurisy}

In several diseases the concentration of lysozyme is often high in body fluids. Raised serum lysozyme levels have been reported in such granulomatous diseases as sarcoidosis, pulmonary tuberculosis, and Crohn's disease. ${ }^{2}$ We have extended studies on the diagnostic significance of lysozyme content in body fluids to the pleural fluid in pleurisy of various aetiologies.

\section{Patients, methods, and results}

The series comprised 37 patients admitted to hospital for the diagnostic evaluation of one-sided or bilateral pleurisy. Patients with malignancies of extrapulmonary tissues were not included. The diagnosis of pleurisy was based on clinical, laboratory, and radiological findings. The patients were divided into three groups according to the final diagnosis: $(a)$ those with tuberculous pleurisy, $(b)$ those with non-tuberculous pleurisy (carcinoma, collagenosis, non-specific pleurisy, and pleural effusion of cardiovascular origin), and (c) those with bacterial empyema. Of the 12 tuberculous patients 10 had either a positive culture for Mycoplasma tuberculosis or a positive pleural biopsy finding, and in two the diagnosis was based on clinical and radiological findings. With EDTA used as anticoagulant, lysozyme activity was determined simultaneously in plasma and in cell-free pleural fluid with the lysoplate method in which purified human lysozyme was the standard. ${ }^{1}$

Lysozyme concentrations in both plasma and pleural fluid were significantly higher in patients with tuberculous pleurisy than in those with non-tuberculous pleurisy (see table). Moreover, the pleural fluid concentration of lysozyme in patients with tuberculosis was significantly higher than the plasma concentration $(P<0.001)$. In 11 out of 12 tuberculous patients but none of the 22 non-tuberculous patients either the concentration of pleural fluid lysozyme was $18.0 \mathrm{mg} / 1$ or greater or the pleural fluid:plasma lysozyme ratio was 1.5 or greater. In three patients with bacterial empyema of the pleural cavity lysozyme activity in pleural fluid was raised considerably. In no group of patients did the lysozyme levels in the pleural fluid correlate either with the total number of cells or with the number of non-lymphoid cells in the pleural effusion.

\section{Discussion}

The culture of $M$ tuberculosis from pleural fluid has been diagnostic in $20-30 \%$ of patients, the culture of pleural biopsy specimens in about $55-80 \%$, and typical histopathological appearances of pleural biopsies in about $60-80 \%$ of patients with tuberculous pleurisy. ${ }^{3}$ Our results, however, show that corroborative additional and specificinformation for the diagnosis of tuberculous pleurisy can be obtained easily and quickly by the determination of lysozyme in pleural fluid. We do not, however, expect the method we used to distinguish between tuberculous pleurisy and pleural effusions associated with sarcoidosis. Because only $10 \mu \mathrm{l}$ of pleural fluid is needed for determining lysozyme activity, this procedure is suitable for studying patients with negligable amounts of pleural effusion.

The origin of lysozyme associated with most inflammatory disorders has been attributed to the accumulation of lysozyme-containing cell types-that is, granulocytes, monocytes, and macrophages-called up by the general inflammatory response. Both in vivo and in vitro the transformation of monocytes into macrophages and epitheloid cells is accompanied by a corresponding increase in the amount of lysosomal enzymes including lysozyme. The microbiocidal power of these cells is progressively increased and mature epitheloid cells have the capacity to destroy tubercle bacili. Thus the possible tuberculostatic effect of lysozyme, the co-factor role of lysozyme inhibiting growth of $M$ tuberculosis, ${ }^{4}$ and the observation that phagocytosis is stimulated by human lysozyme ${ }^{5}$ all strongly suggest that the raised concentrations of lysozyme in the vicinity of tuberculous lesions are of pathogenetic importance.

${ }^{1}$ Osserman, E F, and Lawlor, D P, Fournal of Experimental Medicine, 1966, 124, 921 .

2 Perillie, P E, et al, American fournal of Medical Sciences, 1973, 265, 297

3 Scerbo, J, et al, fournal of the American Medical Association, 1971, 218, 377.

4 Thacore, H, and Willett, H P, Proceedings of the Society of Experimental Biology and Medicine, 1963, 114, 43.

5 Klockars, M, and Roberts, P, Acta Haematologica, 1976, 55, 289.

IV Department of Medicine, Helsinki University Central Hospital, Unioninkatu 38, Helsinki, Finland

M KLOCKARS, MD, assistant physician

T PETTERSSON, $M D$, assistant physician

$\mathrm{H}$ RISKA, MD, assistant physician

Mjölbolsta Hospital, Finland

P-E HELLSTRÖM, MD, assistant chief physician in chest medicine

\section{Muscle cramps, collapse, and seizures in two patients taking metolazone}

Metolazone is a quinazoline-sulphonamide diuretic used in the treatment of hypertension and oedema. Two patients just starting treatment with the drug developed acute muscle cramps followed by impairment of consciousness and epileptiform movements.

\section{Case reports}

A 55-year-old woman had been prescribed metolazone $2.5 \mathrm{mg}$ daily because of a "bloated feeling" She was otherwise healthy and on no other drug. She had been subject to calf-muscle cramps for years. On the third day of treatment she had unusually severe calf-muscle cramps, which recurred suddenly next morning. She collapsed suddenly, remaining unconscious for 10 minutes, during which her husband noted convulsive movements of her left arm and leg. There was no tongue biting or incontinence. She remained drowsy and incoherent for an hour, but physical examination was normal and her blood pressure $120 / 70 \mathrm{~mm} \mathrm{Hg}$. Blood sugar, urea, electrolytes, calcium, cerebrospinal fluid, brain scan, and chest $x$-ray examination were normal. An electroencephalogram was reported as possibly indicative of epilepsy but not diagnostic. The patient had never lost consciousness before and there was no recurrence after discharge.

A 70-year-old man was referred because of resistant oedema and cor pulmonale. Therapy in the first 14 days consisted of daily theophylline, salbutamol, frusemide $160 \mathrm{mg}$, and spironolactone $150 \mathrm{mg}$. Frusemide was then increased to $200 \mathrm{mg}$ daily. Five days later metolazone $5 \mathrm{mg}$ by mouth was prescribed. Twelve hours after this dose he had severe muscle cramps and, minutes later, fell to the ground. Consciousness was impaired, his jaws were tightly clenched, and he was incontinent of urine. The attack lasted two

Mean $( \pm S D)$ lysozyme concentrations $(\mathrm{mg} / \mathrm{l})$ in plasma and pleural fluid of patients with tuberculous and non-tuberculous pleurisy

\begin{tabular}{|c|c|c|c|}
\hline Group & Plasma & Pleural fluid & Pleural fluid:plasma ratio \\
\hline $\begin{array}{l}\text { Tuberculous pleurisy }(n=12) \\
\text { Non-tuberculous pleurisy }(n=22) \\
\text { Bacterial empyema }(n=3)\end{array}$ & $\left.\begin{array}{r}12 \cdot 7 \pm 5.0 \\
9 \cdot 3 \pm 3 \cdot 2 \\
13 \cdot 5 \pm 3.7\end{array}\right\} P<0.025^{*}$ & $\left.\begin{array}{r}23.7 \\
8.3 \pm \begin{array}{r}8.7 \\
371.6\end{array} \\
37.5\end{array}\right\} P<0.001$ & $\left.\begin{array}{r}1.99 \pm 0.7 \\
0.89 \pm 0.2 \\
29.0 \pm 9.7\end{array}\right\} P<0.001$ \\
\hline
\end{tabular}

